\title{
The DELPHI distributed information system for exchanging LEP machine related information
}

\author{
M. Dönszelmann, C. Gaspar
}

\begin{abstract}
An information management system was designed and implemented, whose main purpose is information interchange between the DELPHI experiment at CERN and the monitoring/control system for the LEP (Large Electron Positron Collider) accelerator. This system is distributedand communicates with many different sources and destinations (LEP) using different types of communication. The system itself communicates internally via a communication system based on a publish-and-subscribe mechanism, DIM (Distributed Information Manager).

The information gathered by this system is used for on-line as well as off-line data analysis. It therefore logs the information to a database and makes it available to operators and users via DUI (Delphi User Interface). The latter was extended with the capability of displaying 'time-evolution' plots. It also handles a protocol, implemented using a finite state machine, SMI (State Management Interface), for (semi-)automatic running of the Data Acquisition System and the Slow Controls System.
\end{abstract}

Presented at The International Conference on Accelerator and Large Experimental Physics Control Systems,

Berlin, October 18-22, 1993 

DELPHI (DEtector with Lepton, Photon and Hadron Identification) [1] is one of the four experiments built at LEP (Large Electron-Positron collider) [2] at CERN (European Organisation for Nuclear Research). It consists of a central cylindrical section and two end-caps. The overall length and the diameter are over 10 meters and the total weight is 2500 tons.

The electron-positron collisions take place inside the vacuum pipe in the centre of DELPHI and the products of the annihilations fly radially outwards. The products of the annihilations are "tracked" by several layers of detectors and read out via some 200,000 electronic channels. A typical event requires about 1 million bits of information.

The DELPHI detector consists of 16 subdetectors, each of which records information. This information consists not only of track data, but also of information such as temperatures, pressures, high voltage settings, etc. This huge amount of information is monitored, processed and written on tape. Many parameters, states and settings are also controlled by the Online System. Tapes are eventually used for offline physics analysis.

A system [3] providing an interface between LEP and DELPHI has been running since the start of data taking in August 1989. This system transmits LEP machine information, like bunch current, magnet temperatures and collimator settings to DELPHI. This information is partly needed for monitoring of data taking and partly for offline physics analysis.

The same system also sends DELPHI information, like background levels and luminosities to LEP. The LEP operators use this information to adjust the LEP machine. In future some information will be sent to prevent LEP from doing tuning of the beam if that would be dangerous to DELPHI. The former system, however, suffered from long delays in the transmission of the information and from hangups. It was therefore decided to redesign this part of the Online System.

\section{$2 \quad$ Design}

The new LEP Communication System (LCS) manages information as shown in Fig. 1.

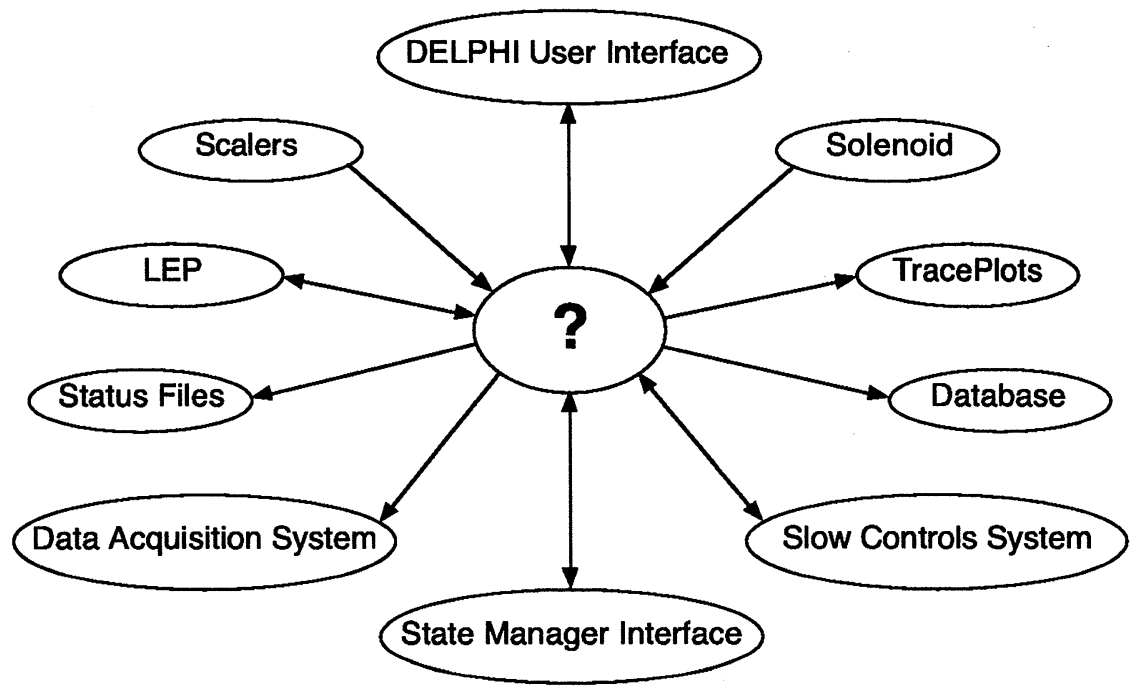

Figure 1: Information Management

A summary of its functionality follows:

- It reads information from LEP via their RPC interface[4].

- It reads DELPHI Scaler[5] information and calculates background levels and luminosities.

- It reads the DELPHI solenoid values via CATS RPC[6].

- It deduces states for some of the available information which it communicates to the State Management Interface[7].

- It keeps information for 'time-evolution' (TracePlot) displays.

- It logs information to a Database[8] via DECNET RPC. 
- It logs information to some Status Files.

- It writes information back to LEP.

- It writes information to the Data Acquisition System[9].

- It writes information to the Slow Controls System[10].

A lot of different communication mechanisms had to be used within the LCS system to transmit all the information from different sources to different destinations. A cascade of timeouts could easily lead to hangups or long delays, if some of the communications would fail.

The LCS system is designed with the following features:

- It fully replaces the previous system with all of its functionality.

- Delays between the sensing of the information and the actual display at LEP or DELPHI are to be kept to a minimum.

- Although communication with several types of protocols is necessary, the system does not hang up completely, but only on the failing protocol.

- It provides easy setup and configuration.

- It provides an easy to use human interface, which includes a facility to view TracePlots.

- It can be distributed over several machines, to avoid cpu overload.

- It interfaces to most other parts of the Online System of DELPHI.

- It is built using existing DELPHI and CERN software components.

\section{Design and Implementation}

The LCS system is designed and implemented under VAX/VMS. Multiple processes provide an event-driven dataflow, using DIM (Distributed Information Management)[11] as an internal communication mechanism. The DUI (DELPHI User Interface)[12] is used as a human interface. An extension to DUI was made to display TracePlots. The SMI (State Management Interface) system is used for control and state monitoring within the LCS system.

\subsection{Multiple Processes}

The LCS system is split up in several processes. Each of those processes use DIM to communicate with the others, and at most one other communication mechanism. If the latter communication fails, for whatever reason, then this process will block while the others continue running. This way only part of the information stream may be blocked.

Instead of using multiple processes one could use multiple threads in one process. This basically gives the same functionality, but at a lower performance cost. VMS provides a package to do this, called DECthreads. However, since DECthreads relies on ASTs (interrupts) for switching between the threads, and many communication mechanisms also heavily use ASTs, this causes clashes and hence cannot be used.

\subsection{Distributed Information Management}

DIM uses a publish-subscribe mechanism and runs on top of DECNET and/or TCP/IP. Any part of the Online System can publish information and any interface can subscribe to this information. The subscriber will be kept up-to-date in an event-driven mode or at regular intervals, to make sure that all subscribers will have the same information at a given moment.

A Name Server handles the client-server communication setup. It keeps track of what runs where and makes it possible to move any of the online parts or any of the interfaces from one machine to another. Every server registers its services (online information) with the Name Server. If a client wants to subscribe to a service, it first contacts the Name Server to find out which server provides that service. It continues by making a direct link with the server, to get the information. The Name.Server also checks for authorisation and recovers lost links.

The LCS system uses DIM for all its internal and some of its external communication. Since DIM provides event- and interval-driven updates, it does not suffer from 'polling' delays.

\subsection{DELPHI User Interface}

DUI provides the user with a Motif interface to the Online System. This interface has a standard layout. To set up different interfaces to different parts of the Online System, DUI is made configurable by means of a simple configuration language. The DUI system merely provides a set of displayable interface blocks, each of which displays one or more items of the Online System. These blocks can be combined, 
by specifying them inside a configuration file, to form a user interface that suits the needs of a particular operator or user. DUI relies on DIM for accessing its information.

DUI was easily reconfigured to include LEP machine related information. An extension was made to provide the display of TracePlots. In the LCS system an extra TracePlot server process was added to keep a 2 hour period of data stored.

\subsection{State Management Interface}

The SMI system handles finite state machines. It is split up in domains, which are connected over DIM. Each of those domains monitors and controls the states of a set of objects. Those objects can belong to other domains or to processes that do actual control and monitoring of some hardware. In DELPHI the SMI system is used to control and monitor almost all parts of the experiment.

In the LCS system SMI is used to control and monitor its states. The states are also communicated to other SMI domains, like the Slow Controls and Data Acquisition Systems, to facilitate in (semi-)automatic running of the DELPHI Online System.

\subsection{Event-Driven Dataflow}

The LCS system uses, for the main part, an event-driven dataflow to make sure that values travel through it with a negligible delay. Fig. 2 shows the flow of information for the data that comes from the scalers. It shows a process, 'Scaler Reader', which does the actual polling over TCP/IP of the scaler hardware. From the moment the data is available in 'Scaler Reader' it will be sent to the 'Background and Luminosity Calculator'. After the calculation is finished, a multi-cast is done to several other processes. Each of those will, upon receipt of the data, act accordingly. Most of these processes are event-driven, meaning they will act as soon as data arrives. Some are event/time driven. They maintain a minimum waiting interval to make sure they will not flood the network.

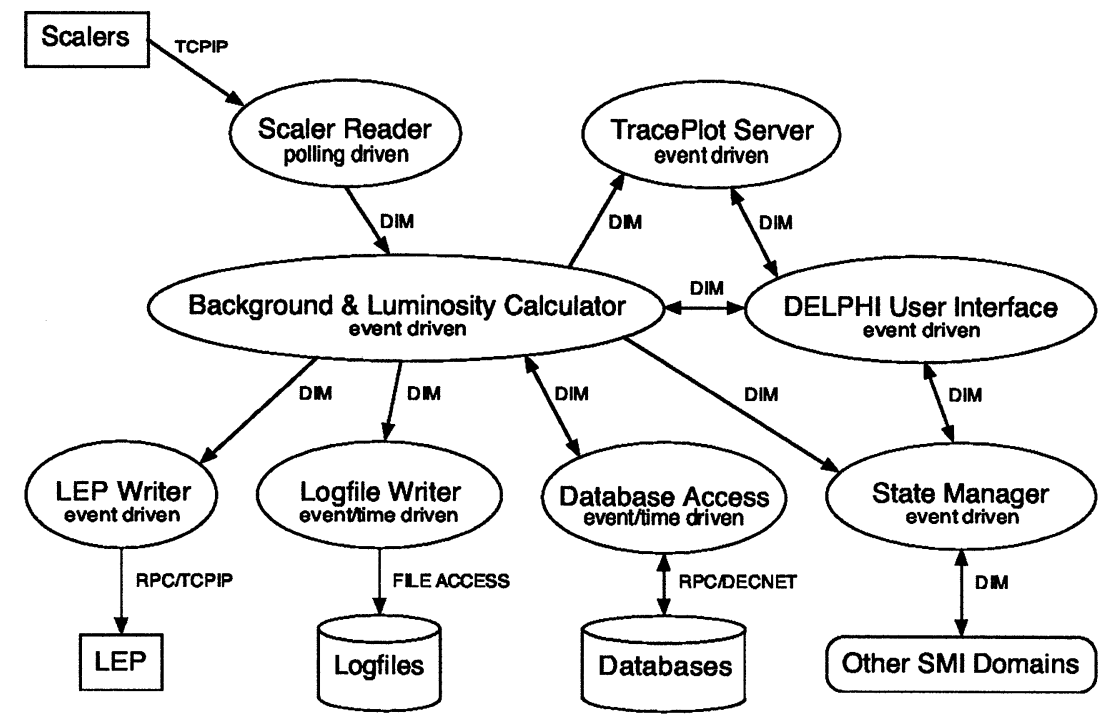

Figure 2: Flow of Scaler Data (Example)

\section{Conclusions}

The design, implementation and use of the LCS system has lead to the following conclusions. It is possible to build a non-blocking communication system by separating the different communication mechanisms into different processes and using one mechanism to communicate between them. An eventdriven communication system is well suited to avoid long delays in updates and to keep information up to date in the system at all times. The use of previously designed systems (e.g. DUI and SMI) to build even larger systems is made easier by defining one interface and communication layer (e.g. DIM). The LCS system is currently in use, providing both DELPHI and LEP with faster updates and more reliable information than with the previous system. 


\section{Acknowledgements}

We would like to thank many members of the DELPHI collaboration who have been very helpful in testing the system described here. In particular we would like to thank A.Grant for informing us on the insights of the previously running system, P.Brummer for providing the software and support on the LEP side, J.Fuster for the details on the trigger and scaler system and Ph.Charpentier for guiding the DAS project.

\section{References}

[1] P.Aarnio et al., The Delphi detector at LEP, DELPHI Collaboration, Nuclear Instruments and Methods in Physics Research A303 (1991) 233-276.

[2] LEP Design Report, CERN/LEP Note 84-01 (1984).

[3] A.Grant, The system for online monitoring of LEP beam background and luminosity at the DELPHI interaction point, CERN/DELPHI Note 93-3 (1993) DAS 137 (to be published).

[4] D.Mathieson, A Programmer's Guide to the LX Remote Communications Package, CERN/LEP Note September (1989).

[5] J.A.Fuster et al., The Online Software for the First and Second Level Trigger of DELPHI, CERN/DELPHI Note 91-112 (1991) DAS 115.

[6] T.Adye et al., Comput. Phys. Commun. 57 (1989) 466.

[7] J. Barlow et al., Run Control in MODEL: The State Manager, IEEE Trans. Nucl. Sci. 36 (1989) $1549-1553$.

[8] Yu.Belokopytov et al., The DELPHI Database Description: LEP Machine Parameter and Luminosity File, CERN/DELPHI Note 93-2 (1993) DAS 136.

[9] T.Adye et al., Architecture and Performance of the DELPHI Data Acquisition and Control System, Proceedings of the International Conference on Computing in High Energy Physics '91, Tsukuba, Japan (1991) 619-626.

[10] T.Adye et al., The Slow Controls of the DELPHI Experiment at LEP, Proceedings of the International Conference on Computing in High Energy Physics '92, Annecy, France (1992) 765-768.

[11] C.Gaspar, M.Dönszelmann, DIM - A Distributed Information Management System for the DELPHI Experiment at CERN, Proceedings of the Eighth Conference on Real-Time Applications in Nuclear, Particle and Plasma Physics, Vancouver, Canada (1993) June 8-11th.

[12] M.Dönszelmann, C.Gaspar, J.A.Valls, A Configurable Motif Interface for the Delphi Experiment at LEP, Proceedings of the International Motif User Conference '92, Washington D.C., USA (1992) $156-162$.

All DELPHI notes given here as reference may be obtained from the authors or from the DELPHI secretariat, CERN, Geneva, Switzerland.

The following are trademarks of Digital Equipment Corporation: Digital, DEC, VAX, VMS and DECthreads. 